\title{
Extracorporeal membrane oxygenation (ECMO) in severe adult respiratory distress syndrome
}

\author{
Peter G. Brindley, MD • Dominic Cave, MD • \\ Laurance Lequier, MD
}

Received: 19 October 2009/Accepted: 27 November 2009/Published online: 15 January 2010

(C) Canadian Anesthesiologists' Society 2009

\section{Article assessed}

Peek GJ, Mugford M, Tiruvoipati R, et al. Efficacy and economic assessment of conventional ventilatory support versus extracorporeal membrane oxygenation for severe adult respiratory failure (CESAR): a multicentre randomised controlled trial. Lancet 2009; 374: 1351-63.

\section{Critical care issue}

Severe adult respiratory distress syndrome (ARDS) remains associated with high mortality despite improved ventilatory techniques. While extracorporeal membrane oxygenation (ECMO) is not uncommon in neonatal/pediatric critical care, studies had yet to show similar benefits in adults. Therefore, the Conventional Ventilation or ECMO for Severe Adult Respiratory Failure (CESAR) Trial by Peek et al. ${ }^{1}$ was eagerly anticipated. It was the first randomized study of ECMO in adult patients in over $15 \mathrm{yr}$ and the largest ever concerning this condition. ${ }^{2}$

\section{Summary and key findings}

In this multicentre randomized control trial from the United Kingdom, 180 patients, aged 18-65 yr, were analyzed. The patients had severe but potentially reversible respiratory failure, a Murray Score of $\geq 3.0$ (determined by combining $\mathrm{PaO}_{2} / \mathrm{F}_{1} \mathrm{O}_{2}$ ratio, positive end expiratory pressure level, lung compliance, chest radiograph appearance, and $\mathrm{F}_{\mathrm{I}} \mathrm{O}_{2}$ of 1.0),

P. G. Brindley, MD $(\bowtie) \cdot$ D. Cave, MD · L. Lequier, MD

University of Alberta, Edmonton, Canada

e-mail: peter.brindley@albertahealthservices.ca or uncompensated hypercapnea $(\mathrm{pH}<7.2)$ despite conventional treatment. They were randomized 1:1 to conventional management without transfer $v s$ transfer to one site and consideration of ECMO. A greater proportion of patients randomized to consideration of ECMO were alive at six months compared with patients randomized to conventional management ( $63 \%$ or $57 / 90$ vs $50 \%$ or $45 / 90$, respectively). This outcome did not reach statistical significance (relative risk $0.73 ; 95 \%$ confidence interval [CI] [0.52-1.03]; $P=0.07)$. However, a significantly greater percentage of patients randomized to consideration of ECMO survived to six months without severe disability compared with patients randomized to conventional management (63\% [57/90] vs $47 \%$ [41/87], respectively; relative risk 0.69 ; $95 \%$ CI [0.050.97]; $P=0.03$ ). Referral for ECMO consideration was associated with 0.03 quality-adjusted years (QALYS) at six months. The cost per QALY for ECMO was 19,252 pounds sterling (GBP) (95\% CI [7,622-59,200]). Despite conventional management, the authors concluded that it was appropriate to transfer adults with severe ARDS to a centre with an ECMO-based management protocol.

\section{Critical appraisal}

Current state of the literature and study relevance

Extra corporeal membrane oxygenation is accepted as a standard-of-care for neonates and children with progressive respiratory and/or cardiac failure refractory to conventional management. ${ }^{1-3}$ In contrast, adult ECMO is currently confined to a few specialized centres and reserved for exceptional cases. This is because of increased cost and logistics but also because of disappointing results from the two previous randomized trials. ${ }^{4,5}$ 
There has been renewed interest in adult ECMO due to encouraging results from case series, ${ }^{6,7}$ improved ECMO technology, ${ }^{2}$ general advances in critical care, ${ }^{3,8}$ and, most recently, the use of ECMO in $\mathrm{H} 1 \mathrm{~N} 1$ patients. ${ }^{9,10}$ In fact, CESAR was published electronically in the same month period as a retrospective case review of ECMO in adult respiratory failure ${ }^{11}$ and a meta-analysis of ECMO following adult cardiac arrest. ${ }^{12}$ The former, a multivariate logistic regression analysis of the Extracorporeal Life Support Organization (ELSO) registry, showed 50\% survival to discharge in 1,473 ECMO patients from 1986 to $2006 .{ }^{11}$ The latter, which encompassed 135 patients from 11 clinical series and nine case reports, found $40 \%$ survived to discharge. ${ }^{12}$ The following month, the aforementioned H1N1 case series from Australasia was released, and it reported approximately $70 \%$ survival to hospital discharge using ECMO for flu-associated ARDS. ${ }^{10}$ In short, CESAR was published at a most propitious time. Even before publication, CESAR was widely debated. ${ }^{3}$ Its publication is already providing considerable fodder for proponents, but also for skeptics. $^{2,8,13}$

\section{Analysis of methodology}

Those randomized to conventional treatment received "best practice" as defined by that treatment centre; no specific protocol was mandated (see below). Those randomized for consideration of ECMO were transferred to a single site, Glenfield Hospital, Leicester. These patients did receive a standardized ARDS treatment protocol, and if they did not respond within $12 \mathrm{hr}\left(\mathrm{F}_{\mathrm{I}} \mathrm{O}_{2}\right.$ still $>90 \%$ or $\mathrm{pH}<$ 7.2), they received cannulation and venovenous ECMO. Seventy-five percent (68/90) of these patients received ECMO. Patients were excluded if they had received peak inspiratory pressures of $>30 \mathrm{~cm} \mathrm{H}_{2} 0$ or $\mathrm{F}_{\mathrm{I}} \mathrm{O}_{2}$ of .0 .8 for $>$ seven days or if they had contraindications to limited heparinization or continued treatment. In other words, the investigators excluded those patients who were unlikely to survive due to lengthy severe ARDS, those who were unable to tolerate ECMO, or those for whom ECMO was inappropriate. Primary outcome was death or severe disability at six months, which was defined as confinement to bed or inability to wash or dress oneself. Secondary outcomes were duration of mechanical ventilation, use of high frequency oscillation/jet ventilation, nitric oxide, prone positioning, steroids, and duration of stay in the intensive care unit (ICU) and hospital. Health status at six months was assessed from an extensive review that included activities of daily living, quality of life, respiratory symptoms, cognitive psychology, and lung function. This assessment included the SF-36 ${ }^{\circledR}$ health survey, EuroQol dimensions, St George's Hospital Respiratory Questionnaire, Hospital Anxiety and Depression Scale, Mini-Mental
State Examination, sleep questionnaire, Caregiver Strain Index, lung spirometry, and upper arm movement. Of note, while patients were not blinded during the initial study period, they were blinded during follow-up. Data from a concurrent British multicentre study of critical care expenditure were used for economic evaluation. ${ }^{14,15}$

Strengths, weaknesses, and external validity

Proponents will likely agree with the authors that CESAR used a "pragmatic" study design (see below) that mirrors the reality of critical care delivery. Furthermore, CESAR showed a significant benefit in the quality of survival $(16 \%$, $P=0.03$ ) using a comprehensive strategy that, in most cases, required ECMO. In contrast, detractors are likely to argue that the lack of blinding during treatment, the use of intention-to-treat analysis, and the absence of standardized protocols in the control group weaken any conclusion. Regardless, all should agree that Peeks et al. deserve credit for undertaking a trial that took years of planning and hundreds of hours to complete. In fact, any debate resulting from CESAR will reflect the complexity (and imperfection) that is typical of trial methodology when studying such critically ill patients.

While the investigators screened 766 patients from 148 centres, only $180(23.5 \%)$ were ultimately enrolled from 68 centres. As such, it is worth emphasizing that this was not primarily a study of all comers with ARDS, nor was it intended to be. However, 108 of the patients who met the criteria for severe ARDS (see above) were excluded simply due to the unavailability of an ECMO bed. In effect, $37.5 \%$ of the study patients were excluded despite meeting criteria. It is impossible to know the number of patients who would have survived without significant disability, and their demographics are not presented. In addition, only three-quarters of the patients who were randomized to consideration of ECMO actually received it. Statistically, significant benefits actually disappear if we remove the patients who were transferred but not treated with ECMO. ${ }^{2}$

External validity is reduced because the benefits could be from transfer to a skilled single ECMO-capable centre rather than from ECMO itself. In contrast to the control group hospitals, Leicester is recognized as a national leader in ARDS treatment. In addition to ECMO access, it possesses a rare combination of knowledge experts, volumebased competence, evidence-based protocolled care, and well-practiced teams. This is likely why hospital staff were able to stabilize 17 of 90 transferred patients so that ECMO was not needed, and over $80 \%$ (14/17) of these survived. Regardless, using a single ECMO-ready centre with atypical resources limits generalizability, even if it reflects a "pragmatic" UK reality. Generalizability may also be in question because the control group survival was lower than 
a multicentre U.S. trial using lower volume ventilatory strategies but no ECMO ${ }^{8}$ and survival in the ECMO group was not substantially better. ${ }^{8}$ Unlike the recent H1N1 series, ${ }^{10}$ the patients were not cannulated prior to transport, and the investigators did not stratify patients into extrapulmonary vs pulmonary ARDS. ${ }^{16}$

The authors claimed that there would have been insufficient support for study enrollment had they demanded that all hospitals use a common protocol. ${ }^{1}$ Regardless, this reduces generalizability. Peeks et al. are to be commended for including the putative dangers of transport (three of 90 patients died prior to transport, and two died during transport) in their analysis. Also, the study design inadvertently highlights how bed shortages can substantially limit access to ECMO and impair critical care research.

\section{Clinical perspective}

Despite the extensive effort put into CESAR, we cannot be sure if other nations or other ECMO-capable hospitals would achieve lower or higher survival results using a comprehensive strategy that includes ECMO. While they have demonstrated economic cost-effectiveness, the authors caution that this is applicable only in settings with similar services to the UK. Presumably, comprehensive economic analysis should also include costs associated with training, maintaining, and equipping a team.

CESAR's design may have been "pragmatic", but it meant that bedside clinicians were not blinded. It is possible that once the considerable resources were invested, i.e., both transfer and initiation of ECMO, that there was a survival advantage as much from reluctance to withdraw as from the salutary effect of ECMO. While this is a methodological criticism, it also offers a clinical reminder of the putative benefit of sheer persistence on survival.

\section{Conclusions}

The CESAR trial demonstrates that an approach to severe adult ARDS that is comprehensive, aggressive, and does not arbitrarily exclude ECMO, can produce results as good as previously obtained ${ }^{4}$ and improve on results from conventional therapy in many centres. Critical appraisal suggests it may also matter where, how, and by whom patients are treated. Survival without severe disability was achieved but at approximately twice the cost and twice the length of stay. ECMO also requires dedicated personnel and equipment not currently available in most ICUs. This landmark study signifies that nations will be forced to decide if its critically ill adult patients deserve this investment, how centralized the centre should be, and how to ensure reasonable access. ${ }^{13}$ Peeks et al. deserve to be congratulated for provoking such a timely and important question.

Conflicts of interest None declared.

\section{References}

1. Peek GJ, Mugford M, Tiruvoipati R, et al. Efficacy and economic assessment of conventional ventilatory support versus extracorporeal membrane oxygenation for severe adult respiratory failure (CESAR): a multicentre randomised controlled trial. Lancet 2009; 374: 1351-63.

2. Zwischenberger JB, Lynch JE. Will CESAR answer the adult ECMO debate? Lancet 2009; 374: 1307-8.

3. Lequier L. Extracorporeal life support in pediatric and neonatal critical care: a review. J Intensive Care Med 2004; 19: 243-58.

4. Zapol WM, Snider MT, Hill JD, et al. Extracorporeal membrane oxygenation in severe acute respiratory failure. A randomized prospective study. JAMA 1979; 242: 2193-6.

5. Morris AH, Wallace CJ, Menlove RL, et al. Randomized clinical trial of pressure controlled inverse ratio ventilation and extracorporeal $\mathrm{CO}_{2}$ removal for adult respiratory distress syndrome. Am J Respir Crit Care Med 1994; 149: 295-305.

6. Kolla S, Awad SS, Rich PB, Schreiner RJ, Hirschl RB, Bartlett $R H$. Extracorporeal life support for 100 adult patients with severe respiratory failure. Ann Surg 1997; 226: 544-64.

7. Peek GJ, Moore HM, Moore N, Sosnowski AW, Firmin RK. Extracorporeal membrane oxygenation for adult respiratory failure. Chest 1997; 112: 759-64.

8. Anonymous. Ventilation with lower tidal volumes as compared with traditional tidal volumes for acute lung injury and the acute respiratory distress syndrome. The Acute Respiratory Distress Syndrome Network. N Engl J Med 2000; 342: 1301-8.

9. Kumar A, Zarychanski, Pinto R, et al. Critically Ill patients with 2009 influenza A (H1N1) infection in Canada. JAMA 2009; 302: 1872-9.

10. Australia and New Zealand Extracorporeal Membrane Oxygenation (ANZ ECMO) Influenza Investigators. Extracorporeal membrane oxygenation for 2009 influenza A (H1N1) acute respiratory distress syndrome. JAMA 2009; 302: 1888-95.

11. Brogan TV, Thiagarajan RR, Rycus PT, Bartlett RH, Bratton SL. Extracorporeal membrane Oxygenation in adults with severe respiratory failure: a multi-center database. Intensive Care Med 2009; 35: doi:10.1007/s00134-009-1661-7.

12. Cardarelli $M G$, Young AJ, Griffith B. Use of extracorporeal membrane oxygenation for adults in cardiac arrest (E-CPR): a meta-analysis of observational studies. ASAIO J 2009; 55: 581-6.

13. White DB, Angus DC. Preparing for the sickest patients with 2009 influenza A (H1N1). JAMA 2009; 302: 1905-6.

14. Thalanany MM, Mugford M, Hibbert $C$, et al. Methods of data collection, analysis for the economic evaluation alongside a national, multi-centre trial in the UK: conventional ventilation or ECMO for severe adult respiratory failure (CESAR). BMC Health Serv Res 2008; 8: 94.

15. Stevens VG, Hibbert $C L$, Edbrooke $D L$. Evaluation of proposed casemix criteria as a basis for costing patients in the adult general intensive care unit. Anaesthesia 1998; 53: 944-50.

16. Gattinoni L, Pelosi P, Suter PM, Pedoto A, Vercesi P, Lissoni A. Acute respiratory distress syndrome caused by pulmonary and extrapulmonary disease. Different syndromes? Am J Respir Crit Care Med 1998; 158: 3-11. 